$L C L S-T N-07-9$

\title{
Summary of QM02 Measurements *
}

\author{
J. Welch, J. Wu
}

September 18, 2007

This note summarizes both the beam-based and various laboratory measurements of quadrupole magnets, units 387 and 428, used for QM02 in the LCLS Injector. These were undertaken because of a consistent discrepancy between accelerator model predictions and beam observations which seemed to indicate a weak QM02. A report 'QM02 Strength Measurement', by Welch and Wu, describes the discrepancy and beam-based measurements on unit 387. Subsequently, unit 387 was replaced by unit 428 , refinements were made to analysis of the beam-based measurements were made, and additional magnetic measurements $^{1}$ were made on unit 387 in the lab. These new results are summarized in this note.

The principle results are:

- Laboratory measurements of integrated gradient for the same magnet, or for different magnets of the same type, are all within $1 \%$ of each other at gradients of interest. These cases cover three independent types of measurements, disassembly/reassembly of the units, and extended periods of time between measurements.

- Standardization, or lack thereof, can cause integrated gradient errors of approximately $0.2 \mathrm{kG}$, which can amount to a few percent of the strength of the magnet depending on the setting.

- Model-independent beam-based measurements indicate the magnets are actually weaker than expected by about 2 percent, but these measurements are subject to the uncertainty of the BPMS1 location.

- The standardization cycle is effective.

- The stainless steel BPM vacuum chamber inside the magnets has no significant effect on the beam.

*Work supported in part by the DOE Contract DE-AC02-76SF00515. This work was performed in support of the LCLS project at SLAC.

${ }^{1}$ The laboratory magnetic measurements were performed by A. Fisher and the authors analyzed the data he provided to come up with the number quoted in this report. 
Table 1: Laboratory and beam-based measurements made on quadrupole magnets used for QM02.

\begin{tabular}{cccll}
\hline \hline Unit & Date & Directory & Devices & Measurements \\
\hline 387 & $5 / 18 / 06$ & 387 & Coil & Integrated gradient \\
387 & $7 / 24 / 07$ & $387 \mathrm{r} 2$ & $\begin{array}{l}\text { Coil, Wire, Hall } \\
\text { Probe }\end{array}$ & $\begin{array}{l}\text { Integrated gradient, field scan, } \\
\text { standardization tests, vacuum } \\
\text { chamber test. }\end{array}$ \\
387 & $5 / 26 / 07$ & & Beam & Integrated gradient \\
\hline 428 & $6 / 21 / 07$ & $428 \mathrm{r} 2$ & Coil & $\begin{array}{l}\text { Re-interpret previous mea- } \\
\text { surements with corrected coil } \\
\text { calibration and reference radius. }\end{array}$ \\
& & & Beam & $\begin{array}{l}\text { Integrated gradient, standardiza- } \\
\text { tion tests. }\end{array}$ \\
\hline \hline
\end{tabular}

The discrepancy between the accelerator model predictions and the actual orbit response is not resolved, but the evidence points away from magnetic strength errors as the source. Differences between the model locations and effective locations of BPM's is a possible culprit. This idea is explored in Section 2.

Table 1 summarizes the measurement activities that were performed on the units. The dates listed correspond to the date when the relevent series of runs was completed.

\section{Integrated Gradient}

The integrated gradient is the line integral of the gradient taken along the beam path, including the fringe fields. In the laboratory it was measured three ways: using a rotating coil, using a translating wire, and using a scanning Hall probe. It was measured in the Injector at low quadrupole current using the electron beam and the focal length method. The results of these measurements are compared for the operational field strength and for the field strength used in the focal length measurement in Table 2. The results are expressed in terms of the current needed to achieve the stated integrated gradient, according to each measurement. To a good approximation the integrated gradient is proportional to current, so relative differences in currents, at the same integrated gradient, are the same as relative differences in integrated gradients, at the same current. For the comparison, currents and integrated gradients were linearly interpolated 
Table 2: Comparison of measurement results of integrated gradient using coils, wires and beam.

\begin{tabular}{ccccc}
\hline \hline Unit & Date & Device & $\begin{array}{c}{[\mathrm{A}] \text { at }-6.300 \mathrm{kG}} \\
\text { (normal op.) }\end{array}$ & $\begin{array}{c}{[\mathrm{A}] \text { at-3.404 kG }} \\
\text { (focal length meas.) }\end{array}$ \\
\hline 387 & $5 / 18 / 06$ & Coil & -3.5589 & -1.8846 \\
387 & $7 / 24 / 07$ & Coil & -3.5888 & -1.9044 \\
387 & $5 / 26 / 07$ & Beam & & -1.9983 \\
\hline 428 & $6 / 21 / 07$ & Coil & -3.5921 & -1.9055 \\
428 & $7 / 7 / 07$ & Beam & & -1.9417 \\
\hline \hline & \multicolumn{5}{c}{$[\mathrm{A}]$ at $-6.3336 \mathrm{kG}$} \\
\hline 387 & $5 / 18 / 06$ & Coil & -3.5873 & \\
387 & $7 / 24 / 07$ & Wire & -3.5840 & \\
387 & $7 / 24 / 07$ & Coil & -3.6083 & \\
387 & $7 / 24 / 07$ & Hall & -3.6120 & \\
\hline 428 & $6 / 21 / 07$ & Coil & -3.6131 & \\
\hline \hline
\end{tabular}

from measured data. The Hall probe data was extrapolated from the measured integrated gradient of $6.247 \mathrm{kG}$. There is a small difference, typically a few tenths of a percent or less, between currents interpolated in this way and currents that are calculated using the polynomial fit that was put into the database.

The measurements on unit 387 with the Coil, Wire and Hall probe at $-6.3336 k G$ give currents that are all within a total range of less than $1 \%$. Comparison of Coil measurements of unit 387 and unit 428 indicate that at the operating current the two units have the same strength to within $1 \%$.

There is significant a difference between the focal length measurement result and the results from measurements made in the lab. For unit 387 this beam based measurement indicates the magnet is 5 or $6 \%$ weaker, depending on which Coil measurements is compared. However, it should be noted that hystersis was not accounted for in the beam based measurement of unit 387, so it may or may not have contributed to the observed difference. Hystersis was accounted for in the beam-based measurement of unit 428. In this case the beam based measurement indicates the unit is $2 \%$ weaker than the Coil measurement indicates. Figure 1 plots the theoretical value of integrated gradient needed for perfect focus along with the 'polynomial' value which is derived from laboratory measurements and the value of the measured current needed to get perfect focus. Ideally the integrated gradient and current lines would intersect on the polynomial for the upward branch. Coincidentally?! there is perfect agreement between the decreasing branch from magnetic measurements and the focal point measurement when the unit was deliberating de-standardized. 
QM02 Replacement (\#428)

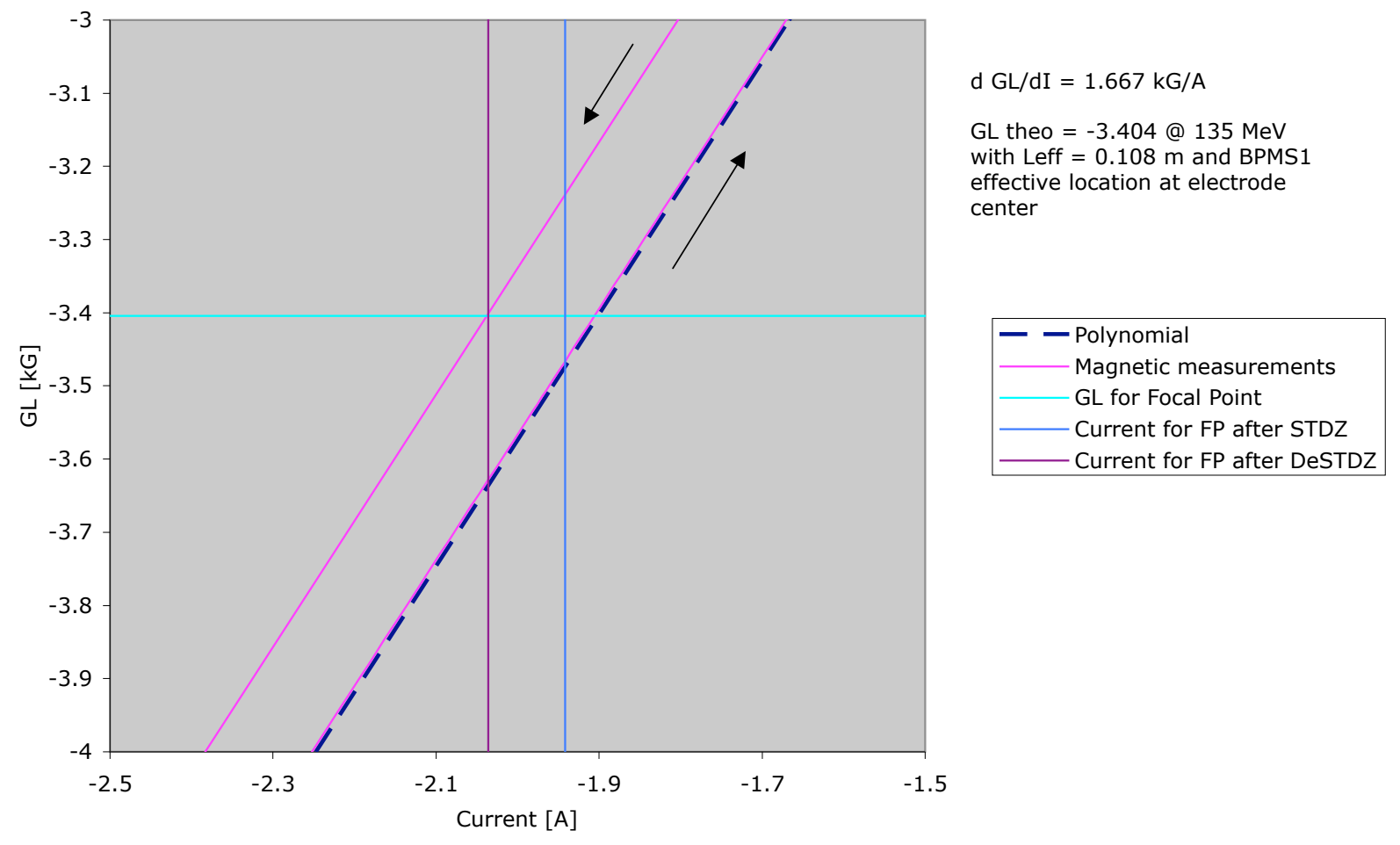

Figure 1: Comparisons of focal point and rotating coil based measurements of integrated gradient. 


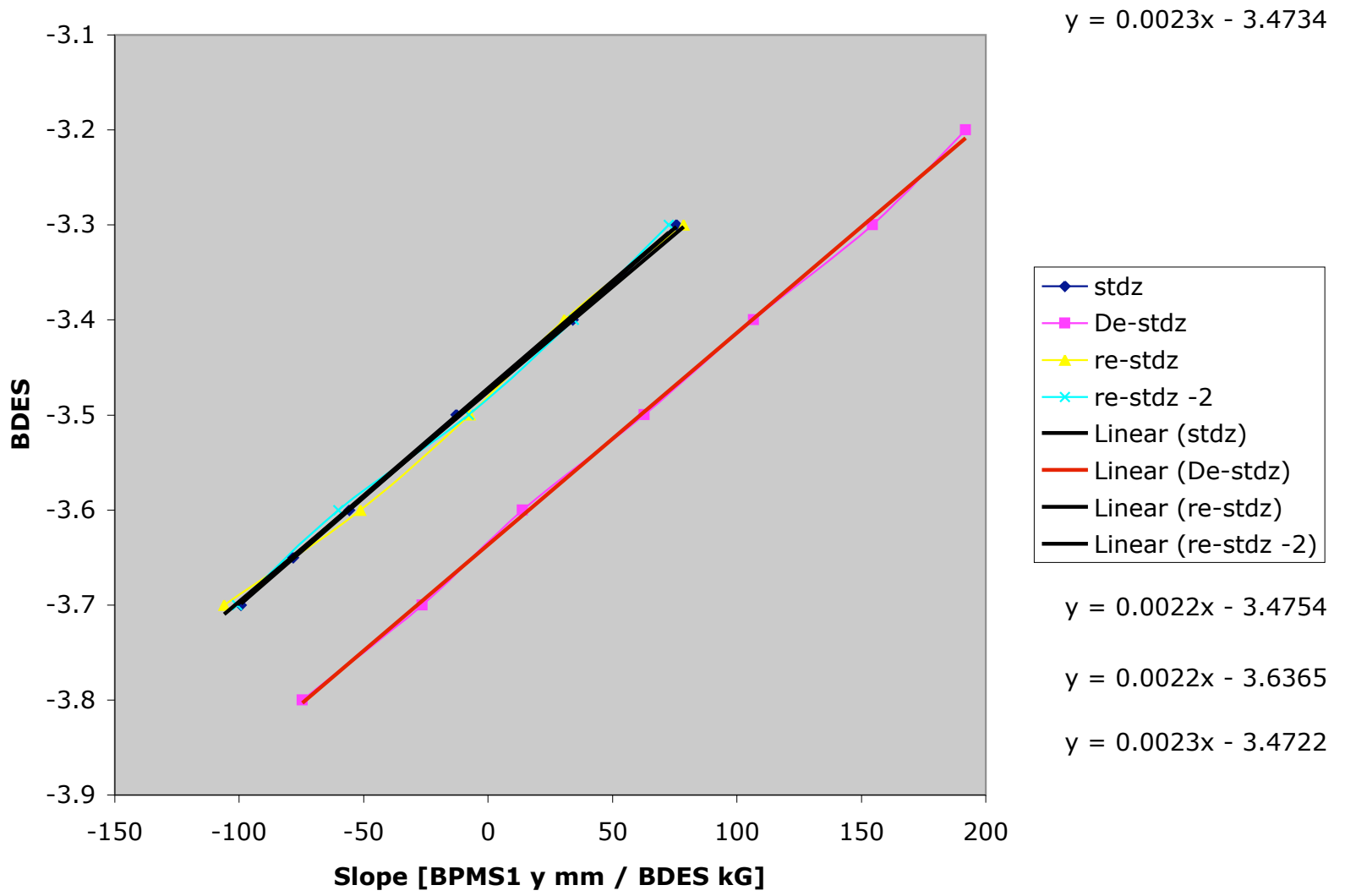

Figure 2: Effects of hystersis on beam response to corrector change.The slope of the change in the beam position versus the change in the corrector strength is a measure of how far QM02 is from perfectly focussing the deflection of the beam by corrector YC07 onto BPMS1. When the slope equals zero, there is no vertical motion seen on BPMS1 due to a change the strength of corrector YC07. The equations for the lines fitting the data are listed and the intercepts give the best estimate of the focal strength of QM02 needed for a perfect focus. The method is quite sensitive and reproducible. The largest uncertainty is in the effective location of the BPMS1. 


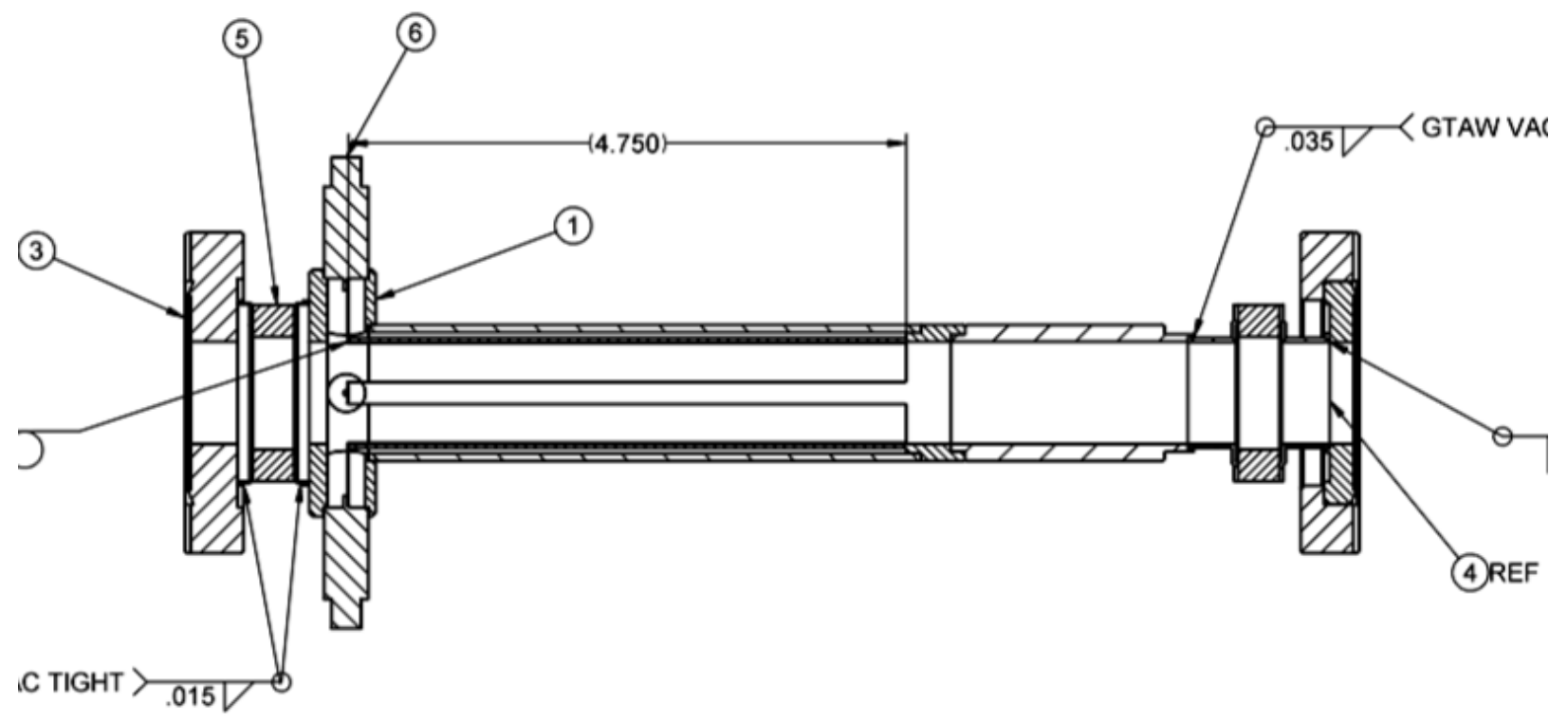

SECTION A-A

Figure 3: Section of the vacuum subassembly containing the electrode of the BPMs. This subassembly is used in many places in the Injector.

\section{BPM Location}

In the model of the injector each quadrupole is split exactly in the middle and a zero-length BPM is located between the two halves. On the other hand, in reality the effective location of the BPMs is somewhat different. BPMs were built into a vacuum subassembly and designed to have the mechanical center of the full subassembly containing the BPM electrodes to be located at the center of the quadrupole. See Figure 3. Since the BPM electrodes are offset from the mechanical center of the vacuum subassembly they were not placed in the center of the quadrupole. Furthermore the effective location of the BPM may not be the center of the electrodes, but depends on the signal processing and may be further toward the feedthroughs. In addition, further uncertainty arises because bellows on each end of the subassemblies allow some freedom in the installed locations.

Normally, this degree of uncertainty in the effective position of the BPMs is not important. However the focal length of QM02 is short, about $0.7 \mathrm{~m}$, and uncertainties in the BPM location of order $0.05 \mathrm{~m}$ are possible given the known offsets and uncertainties in effective location. So differences can be expected of order several percent in elements of the transfer matrix, between the model 


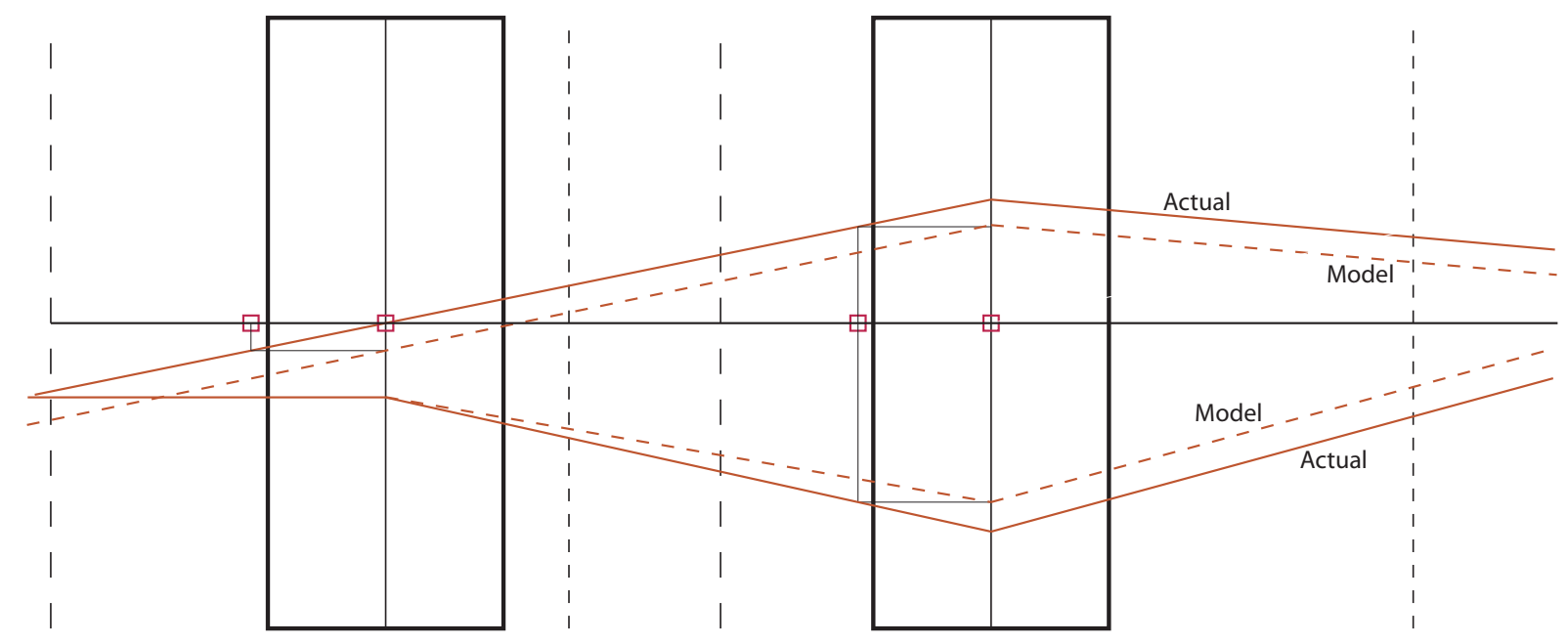

$\mathrm{QM} 01, \mathrm{f}=0.307 \mathrm{~m}$

$\mathrm{QM} 02, \mathrm{f}=-0.387 \mathrm{~m}$

for horizontal focussing

$\square$ BPM

Vertical plane shown

scale 1 inch $=0.14$ meters

Figure 4: In the region of QM01 and QM02, rays traces are shown by solid line and what the Model sees is in dashed line for the vertical plane.

of the accelerator and the actual performance. In the case of the focal length measurement, if the effective location of the BPM is actually at the feedthrough location, which is on the upstream end of the vacuum subassemblies, the model would not be in disagreement with the magnetic measurement results.

The effect of the erroneous BPM locations can be seen graphically in Figures 5 and 4. For both figures, the QM01 and QM02 quadrupoles are drawn to-scale horizontally and the principle rays through QM01 are traced. The real path of the ray is shown in solid line, while the path the model interprets based on the erronous BPM position is shown in dashed lines. Evidently there is a substantial disagreement. Detailed analysis has yet to be done, to determine which, if any, real positions of the BPMs could explain the observed data. One thought is that by increasing the strength of QM02, the actual (virtual) image seen downstream of QM02 can be shifted onto the location that the model thinks it is at, thereby making the model fit the data. 


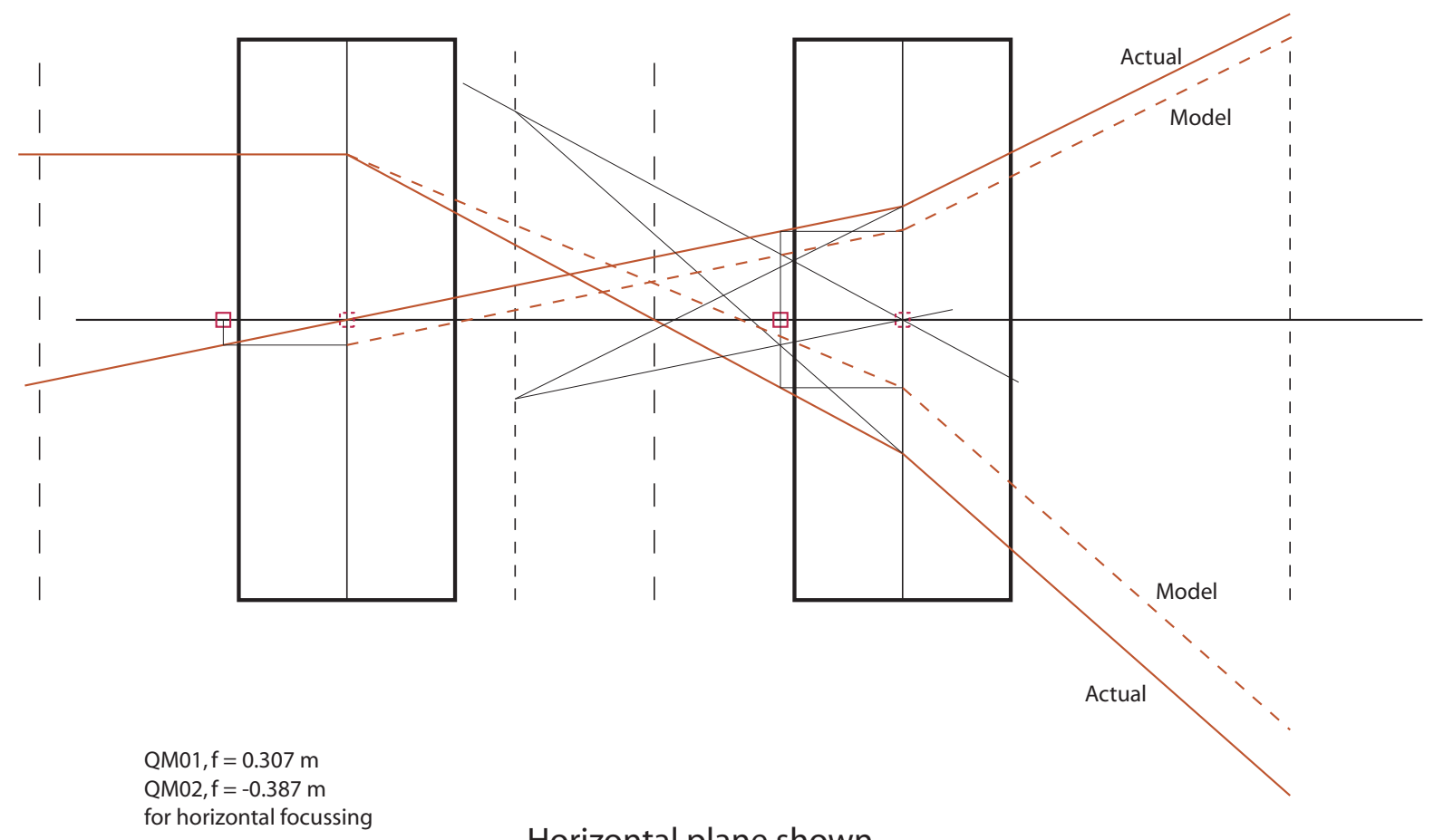

$\square$ BPM

Horizontal plane shown

scale 1 inch $=0.14$ meters

Figure 5: In the region of QM01 and QM02, rays traces are shown by solid line and what the Model sees is in dashed line for the horizontal plane. 
An integrated gradient of $-3.404 \mathrm{kG}$ is assumed for the focal length measurement. This is based on the Mathematica analysis of a thick lense quadrupole, with effective length of $0.108 \mathrm{~m}$ and an effective position of the BPMS1 at the midpoint of the electrode. The current listed in Table 2 is the value of the QM02 current that was required to get the corrector YC07 focussed on BPMS1. The largest uncertainty in this measurement is in knowledge of the effective BPM position.

\section{Effective Length}

Hall probe scans were made on unit 387 at $3.578 \mathrm{~A}$ and at $-12.018 \mathrm{~A}$. Both measurements yielded an effective length of $0.1062 \mathrm{~m}$. The model value for the effective length was $0.108 \mathrm{~m}$. Effective length is defined as the integrated gradient divided by the gradient at the center of the quadrupole. The Hall probe data consisted of a few $X Y$ scans. The gradient was calculated numerically to second order from scans at $Y=0.005, Y=0.000$ and $Y=-0.005$. The integrated gradient was calculated by numerically integrating along the $X$ coordinate.

The Mathematica model of the focal point measurement described in the 'QM02 Strength Measurement' report was run with different effective lengths. The difference in required integrated gradient to get the beam to focus on BPMS1 due to a change from $0.108 \mathrm{~m}$ to $0.1062 \mathrm{~m}$, was completely insignificant.

\section{Standardization Tests}

By convention, the operational state of the quadrupoles should be arrived at by going through a standardization procedure which ends with the magnet current at its minimum (in some cases, the most negative value). Following the procedure, the operational state is reached by raising the current to the design value without overshooting. The standardization procedure was designed to obtain reproducible fields that are not greatly influence by the past magnetic history (hysteresis). Over the operational range of the QM02 magnet, laboratory measurements yield curves of integrated gradient versus current on the standardization cycle for increasing and decreasing currents, that are approximately parallel with an offset of about $0.23 \mathrm{kG}$. (See Figures 2 and 1.) For the beam-based focal length measurement, this difference amounts to a potential error of $6.4 \%$. At the design strength of $6.28 \mathrm{kG}$ hystersis can amount to a potential error of $3.7 \%$.

A beam-based test of the standardization of unit 428 was performed using the focal length method and the results are given in Figure 2. Four measurements were made where the strength of unit 428 was varied and the ratio of the change in vertical position of the beam in BPMS1 with respect to change in the vertical corrector $\mathrm{YC} 07$ strength were recorded. Zero ratio means that the YC07 corrector is exactly imaged onto BPMS1 by unit 428 so that all vertical 
Table 3: Effect of stainless steel vacuum chamber on integrated gradient for unit 387 .

\begin{tabular}{lc}
\hline \hline Case & GL [kG] at -3.584 A \\
\hline With vacuum chamber & -6.3336 \\
Without vacuum chamber (1) & -6.3235 \\
Without vacuum chamber (2) & -6.3140 \\
\hline \hline
\end{tabular}

angles converge to the same vertical position. In three of the measurements unit 428 was standardized. The average BDES of standardized measurements was $3.4736 \pm .0016 \mathrm{kG}$; the standard deviation was only $0.05 \%$ of the average. For one measurement unit 428 was deliberately de-standardized by ramping the current up to the maximum of $12 \mathrm{~A}$ and then down to the desired value. In this state the BDES required for focussing was -3.6365 - about $4.7 \%$ higher than in the standardized state. Thus standardization of unit 428 is effective in accurately re-establishing the integrating gradient, while failing to do can result in significant weakness.

\section{Effect of Stainless Vacuum Chamber}

In Table 3 are the results of wire measurements made on unit 376 with and without a substitute stainless steel vacuum chamber which included BPM electrodes. These measurements were undertaken to see if there was any change of the integrated field due to magnetic properties of the stainless steel. The second data set for "Without vacuum chamber (2)" is just a measurement repetition after a standardization cycle. Nominally there should be no difference between the two measurements made without the vacuum chamber. The measured difference is about $0.01 \mathrm{kG}$, which is comparable to the measured difference between the "With vacuum chamber" measurement and the measurements without the vacuum chamber. Therefore, to within the measurement precision, the data indicate that the presence, or absence, of a the stainless steel BPM chamber has no significant effect on the integrated gradient. 